\title{
P16-specific DNA methylation by engineered zinc finger methyltransferase inactivates gene transcription and promotes cancer metastasis
}

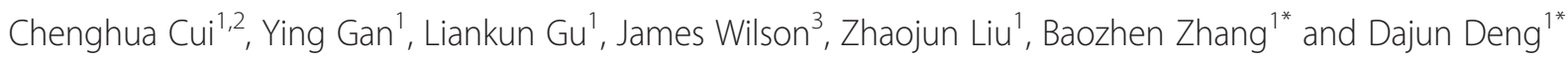

\begin{abstract}
Background: P16 DNA methylation is well known to be the most frequent event in cancer development. It has been reported that genetic inactivation of P16 drives cancer growth and metastasis, however, whether P16 DNA methylation is truly a driver in cancer metastasis remains unknown.

Results: A P16-specific DNA methyltransferase (P16-dnmt) expression vector is designed using a P16 promoter-specific engineered zinc finger protein fused with the catalytic domain of dnmt3a. P16-dnmt transfection significantly decreases P16 promoter activity, induces complete methylation of P16 CpG islands, and inactivates P16 transcription in the HEK293T cell line. The P16-Dnmt coding fragment is integrated into an expression controllable vector and used to induce P16-specific DNA methylation in GES-1 and BGC823 cell lines. Transwell assays show enhanced migration and invasion of these cancer cells following P16-specific DNA methylation. Such effects are not observed in the P16 mutant A549 cell line. These results are confirmed using an experimental mouse pneumonic metastasis model. Moreover, enforced overexpression of $P 16$ in these cells reverses the migration phenotype. Increased levels of RB phosphorylation and NFkB subunit P65 expression are also seen following P16-specific methylation and might further contribute to cancer metastasis.
\end{abstract}

Conclusion: P16 methylation could directly inactivate gene transcription and drive cancer metastasis.

Keywords: Cancer, Engineered methyltransferase, Metastasis, Methylation, P16

\section{Background}

P16 (CDKN2A or Ink4a) is one of the most frequently deleted genes in cancer genomes and has been studied extensively [1]. P16 germline mutation carriers have been shown to have a greatly increased predisposition to familial melanoma [2-4]. Recently, genetic inactivation of $P 16$ has been proven to be a driver for cancer metastasis in mice [5].

While genetic alterations in P16 do occur, gene methylation is far more prevalent in human cancers [6-10]. Studies have shown that P16 DNA methylation is

\footnotetext{
*Correspondence: zbz94@126.com; dengdajun@bjmu.edu.cn Chenghua Cui and Ying Gan are co-first authors.

'Key Laboratory of Carcinogenesis and Translational Research (Ministry of Education/Beijing), Department of Aetiology, Peking University Cancer Hospital \& Institute, Beijing 100142, China

Full list of author information is available at the end of the article
}

correlated with a decreased level of expression in tissues [6-10] and is linked to the development and metastasis of many cancers [11-15]. It is therefore highly likely that P16 DNA methylation may play an important role in cancer development.

It has been reported that artificial P16 DNA methylation induced through the insertion of alu motifs increased the susceptibility of mice to developing cancer [16]. However, whether P16 DNA methylation drives cancer metastasis has not been characterized. In the present study, a P16-specific DNA methyltransferase (P16-dnmt) was used to directly inactivate P16 transcription and the subsequent effects on proliferation, migration, and invasion of cancer cells were evaluated in vitro. These results were further confirmed in immuno-deficient mice. This study provides experimental evidence that 
strongly implicates P16 DNA methylation as a driver in cancer metastasis.

\section{Results}

P16 DNA methylation directly inactivates gene transcription

In order to determine whether P16 DNA methylation directly inactivates gene transcription, a $P 16$ promoterspecific DNA methyltransferase $(P 16-d n m t)$ was initially constructed using the pcDNA3.1_myc/His vector as described in the methods section (Fig. 1a). Western blot analysis confirmed that endogenous P16 was greatly reduced in HEK293T cells $48 \mathrm{~h}$ following transient transfection with the P16-dnmt vector (Fig. 1b). A dual-luciferase reporter assay further illustrated that P16 promoter activity was significantly inhibited in the P16-dnmt-transfected cells (Fig. 1c). Notably, methylation of $\mathrm{CpG}$ islands within both the P16 promoter and exon-1 regions was detected using denatured high performance liquid chromatography (DHPLC) and bisulfite-sequencing (Fig. 1d and e). An additional control lacking approximately $80 \%$ of the DNA methyltransferase activity (R882H mutant) was constructed to evaluate the impact of steric hindrance from P16-Dnmt DNA binding on gene transcription. As expected, chromatin-immunoprecipitation (ChIP)-PCR analysis showed that the mutant still bound with the P16 promoter DNA fragment (Fig. 2a), but did not induce P16

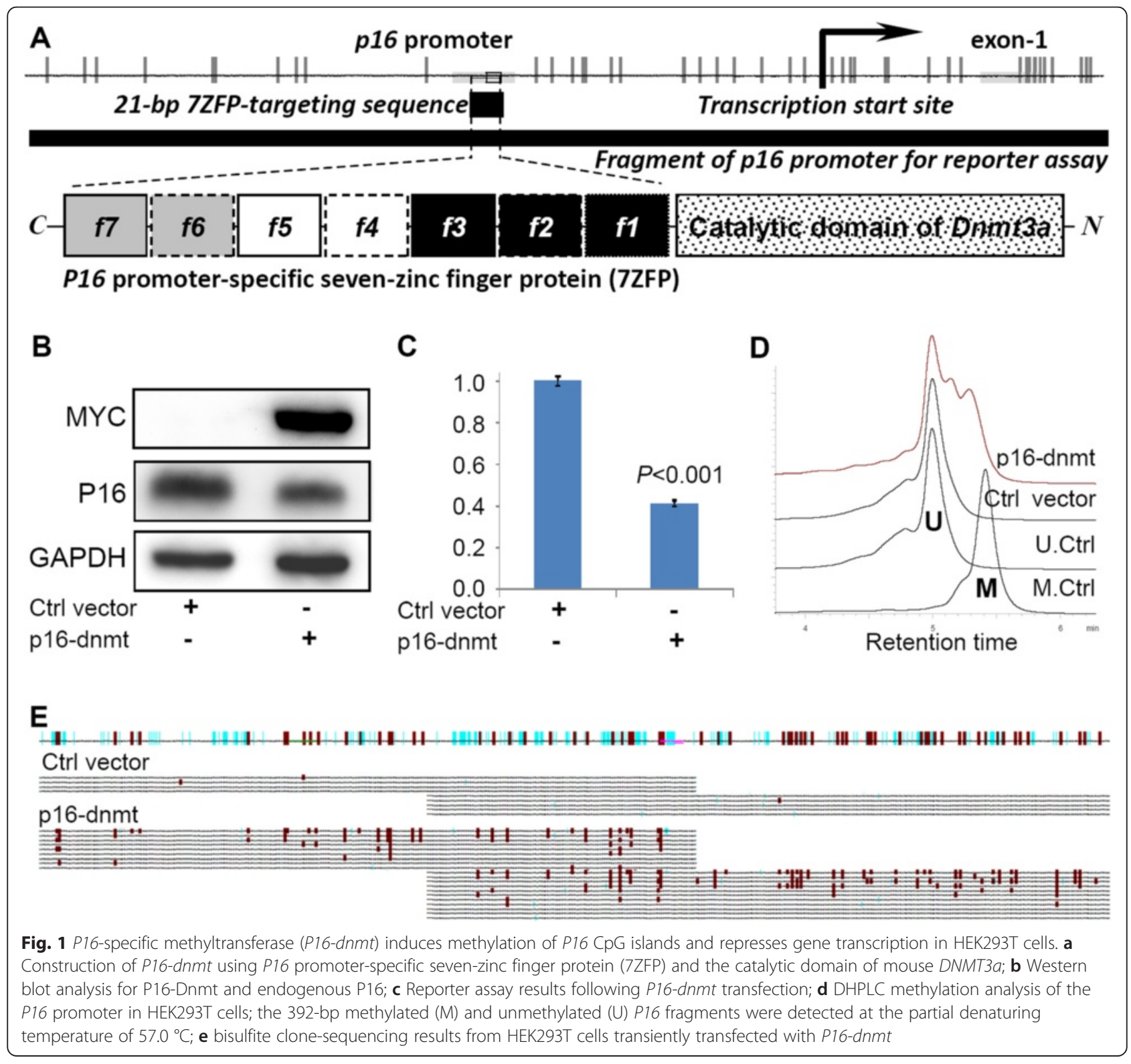



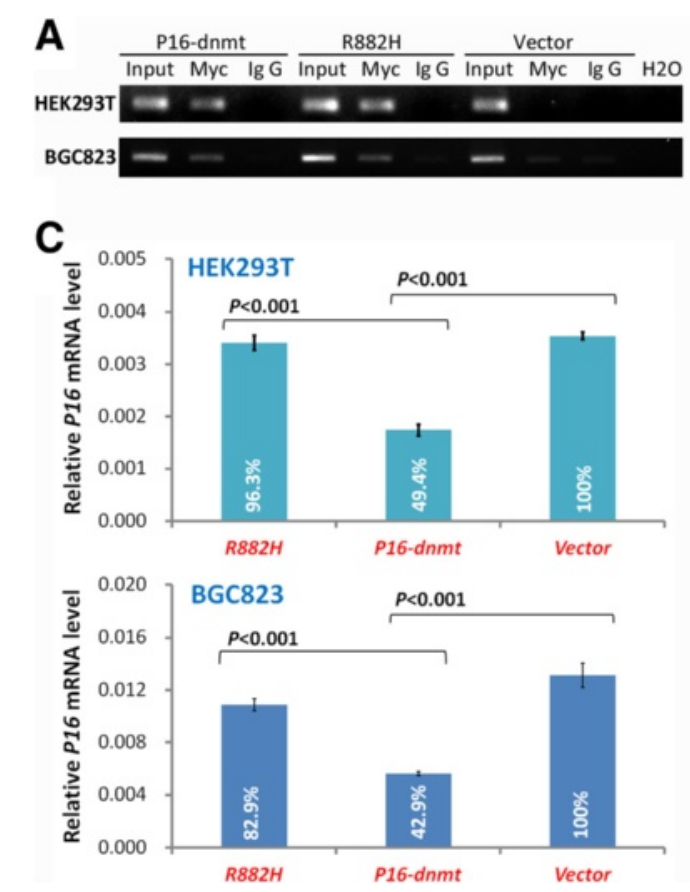

Fig. 2 Comparison of DNA binding capacity, DNA methylation induction, and P16 expression levels between P16-dnmt and the R882H mutant in HEK293T and BGC823 cells $72 \mathrm{~h}$ following transient transfection. a Chromatin-immunoprecipitation (ChIP)-PCR results comparing P16 promoter DNA binding for P16-Dnmt and R882H protein; b Methylation-specific PCR (MSP) detecting methylated and unmethylated P16 alleles; c Quantitative RT-PCR indicating P16 mRNA levels; d Western blot of P16 and Myc/P16-Dnmt protein levels

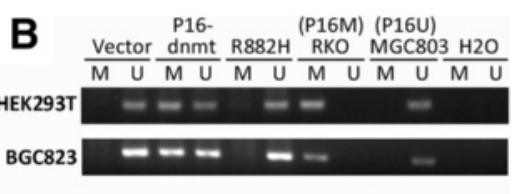

D
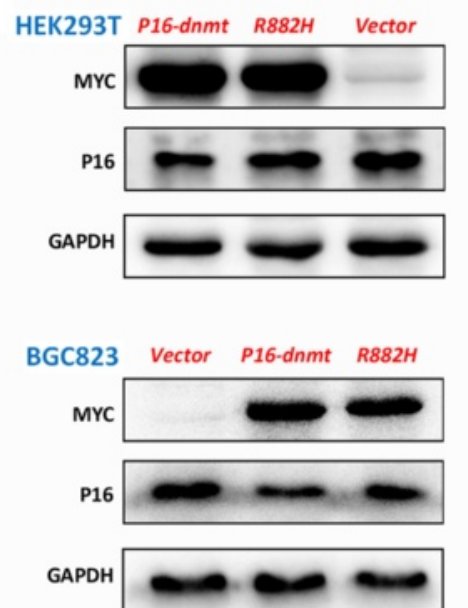

DNA methylation (Fig. 2b). Furthermore, its capacity to repress P16 expression was sharply decreased in both HEK293T and BGC823 cells (Fig. 2c and d). These data suggest that P16 DNA methylation is directly responsible for $P 16$ repression as opposed to steric hindrance. Taken together, these results indicate that P16-dnmt encodes an active methyltransferase for P16 CpG islands, and P16 DNA methylation is sufficient to inactivate endogenous P16 expression.

In order to specifically methylate P16 CpG islands, the P16-dnmt coding-sequence was then integrated into the pTRIPZ lentivirus vector carrying a 'Tet-on' switch to allow the gene expression to be controlled. Expression of P16-Dnmt protein was induced in GES-1 cells stably transfected with the P16-dnmt pTRIPZ vector after treatment with $0.25 \mu \mathrm{g} / \mathrm{mL}$ doxycycline for 3 days (61KD; Fig. 3a). Significant inhibition of endogenous P16 expression was observed in Western blot and quantitative RT-PCR analysis when compared to GES-1 cells transfected with the dnmt3a and $7 Z F P$ control vectors (Fig. 3a and b). Confocal microscopy revealed that the average density of nucleic P16 gradually decreased in the P16-dnmt expressing cells (Fig. 3c). In fact, after treatment with doxycycline for 3 and 7 days, P16 expression levels were decreased by $21.4 \%$ and $53.3 \%$, respectively $(P<0.001)$. Most importantly, intensive methylation of $P 16 \mathrm{CpG}$ islands was induced in the GES-
1 cells stably transfected with P16-dnmt and treated with doxycycline, but not in cells transfected with the control vectors, nor in cells that did not receive doxycycline treatment (Fig. 3d). Similarly, P16 DNA methylation and subsequent repression of P16 expression was also induced by P16-Dnmt in the BGC823 cell line (Additional file 1: Figure S1).

ChIP-PCR analysis also showed that P16-Dnmt specifically bound the P16 promoter, but not the P14 promoter (Additional file 1: Figure S2). Similarly, ChIP-sequencing confirmed that the P16-Dnmt binding fragment was only detected in the promoter of P16-Dnmt/Myc antibody immunoprecipitated DNA from the P16-dnmt-expressing BGC823 cells, but not in the IgG control, nor the cells transfected with the control vector (Fig. 4a, red fragment; Additional file 2: File S1, Additional file 3: File S2, and Additional file 4: File S3). Although most of the P16-Dnmt binding fragments were found in intergenic and intron sequences (Additional file 1: Figure S3A), the main P16-Dnmt binding motif was found to closely match the antisense strand of the targeted fragment in the P16 promoter with a similarity of $21 / 23$ (91.3\%) base pairs (Additional file 1: Figure S3C, red-framed motif). Genome-wide methylation analysis of P16-Dnmt expressing BGC823 cells was performed using an Infinium Methylation $450 \mathrm{~K}$ array. The results illustrated that 647 of 481,615 informative CpG sites 

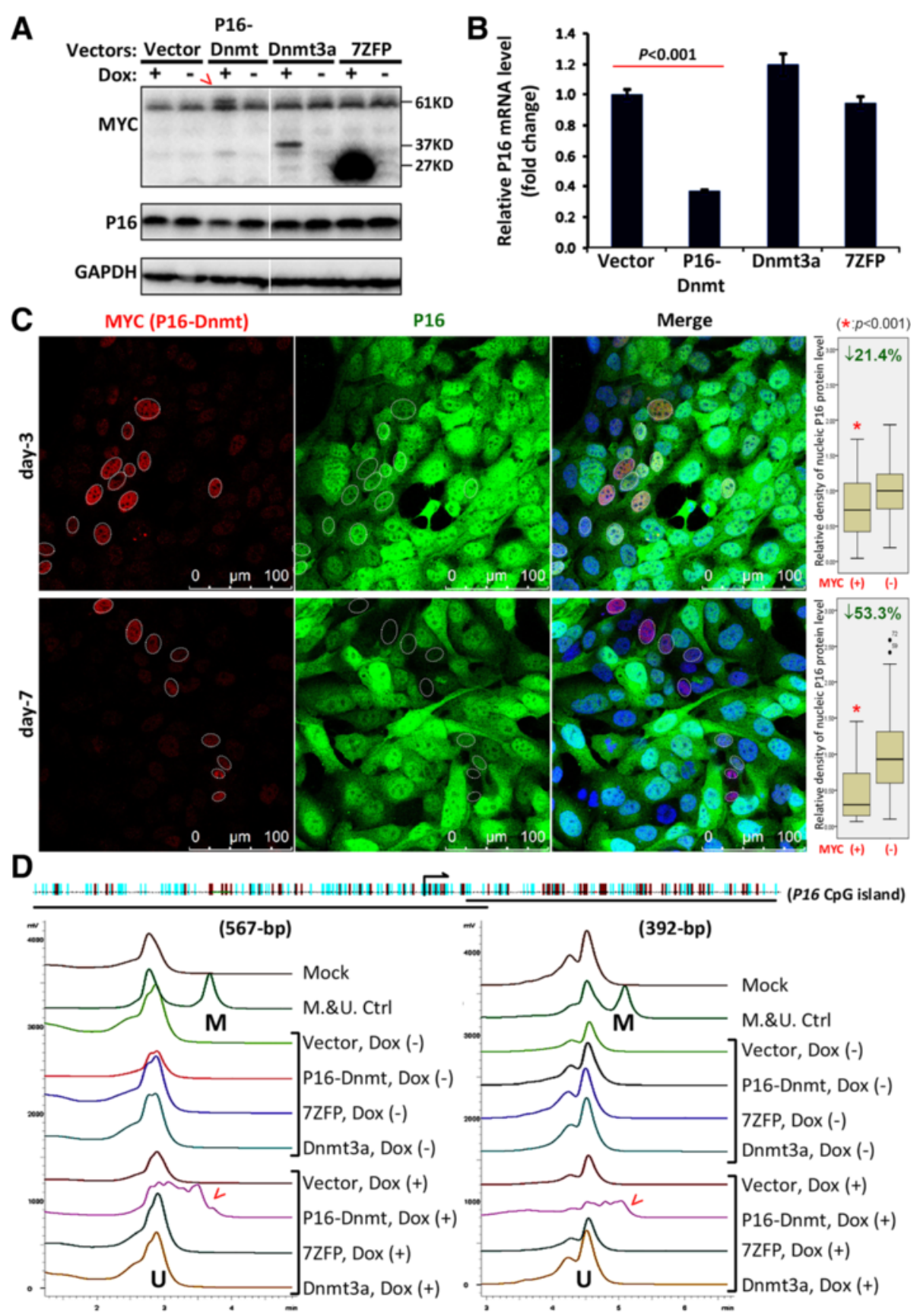

Fig. 3 P16 DNA methylation analysis of GES-1 cells stably transfected with the P16-dnmt pTRIPZ Tet-on' vector. a Western blot analysis of P16 and P16-Dnmt (61 KD, arrow) and control vectors following treatment with $0.25 \mu \mathrm{g} / \mathrm{mL}$ doxycycline for 3 days. b Quantitative RT-PCR results for the P16-dnmt stably transfected, doxycycline-treated cells. c Confocal analysis with P16 labeling following 3 and 7 days of doxycycline treatment. d Confirmatory DHPLC methylation analysis of P16-specific methyltransferase expressing cells (arrow) and controls following 7 days of doxycycline treatment. The 567-bp methylated (M) and unmethylated $(U)$ P16 promoter fragments were analyzed at the partial denaturing temperature of $54.0^{\circ} \mathrm{C}$. DNA samples from HCT116 cells containing both the methylated and unmethylated P16 alleles were used as controls (M.\&U.Ctrl)

$(0.13 \%)$ were significantly hypermethylated $(\Delta \beta>0.50)$. Interestingly, 229 of these $647 \mathrm{CpG}$ sites were located in intragenic $\mathrm{CpG}$ islands and shores corresponding to 203 genes (Additional file 5: File S4). The targeted P16 CpG island was included in the list of differentially hypermethylated sites (Fig. 4a, blue arrow). Furthermore, DNA methylation was not induced in the CpG islands of two control genes, P14 (located within the same CDKN2A locus as P16) and ZNF382 (located on a different chromosome) (Fig. 4b). These results suggest 


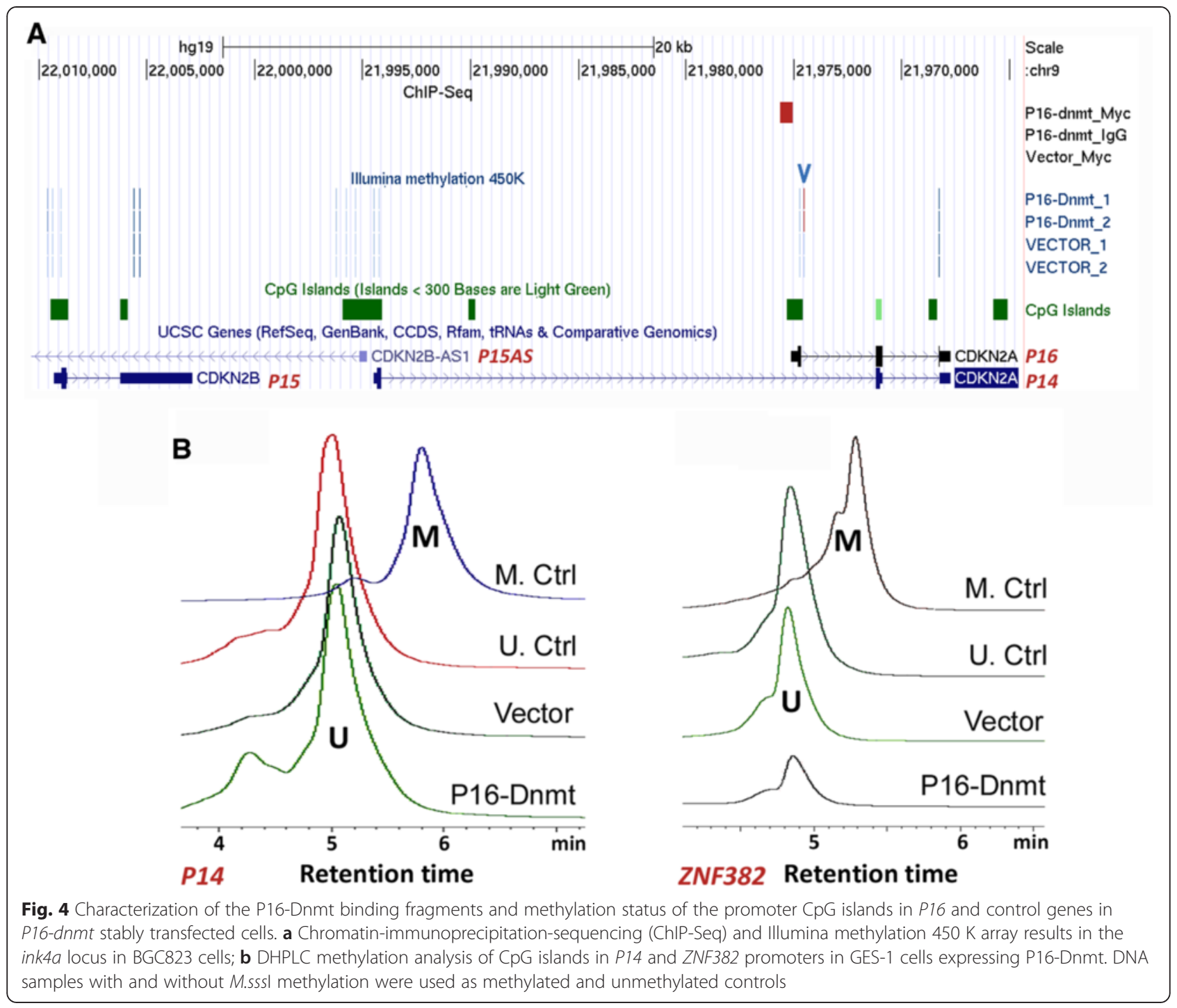

that doxycycline-induced P16-dnmt expression could specifically methylate P16 CpG islands.

\section{P16-specific DNA methylation promotes migration and invasion of cancer cells}

Various assays were then conducted to further characterize the biological behaviors of cancer cells following P16-specific inactivation by DNA methylation. Transwell assays revealed that the migration ability of GES-1 and BGC823 cells was significantly increased following P16-specific DNA methylation (Fig. 5a and b). Similarly, Matrigel assays showed that the invasion capacity of these cell lines was also significantly enhanced by P16-specific DNA methylation (Fig. $5 \mathrm{c}$ and d).

Four weeks after BGC823 cells stably transfected with P16-dnmt were injected into the tail vein of the NOD SCID mice, metastatic nodules were observed in the lung (Fig. 6a). The average lung weight, which correlates with the number of metastatic cells, in the P16-dnmt group was $152.5 \%$ that of the empty vector control group (Mann-Whitney test, $P<0.001$; Fig. $6 \mathrm{~b}$ ). The average proportion of metastatic nodule area to total lung area in the P16-dnmt group was also significantly higher than the control group $(P<0.004$, Fig. $6 c)$.

In addition, P16-specific DNA methylation was found to slightly, but significantly, inhibit proliferation of GES-1 cells, while the proliferation of BGC823 cells was not affected (Additional file 1: Figure S4). However, growth inhibition of the P16-dnmt transfected GES-1 cells was not observed in the SCID mice despite detection of methylated-P16 alleles in the xenografts (Additional file 1: Figure S5).

In order to confirm whether the enhanced migration of cancer cells is P16 DNA methylation-specific, a rescue 


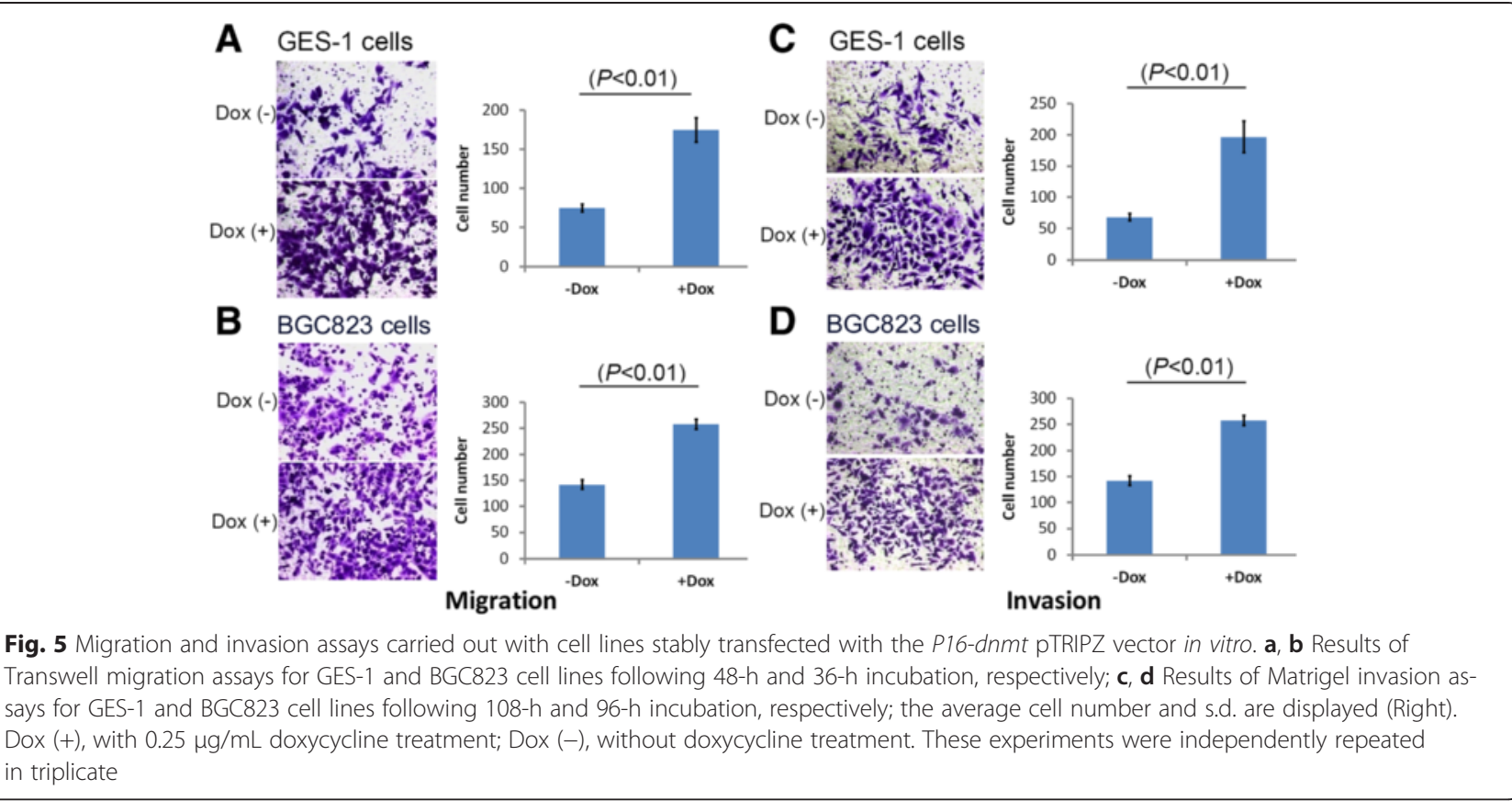

assay was carried out in the P16-dnmt expressing BGC823 cells through transient transfection of a P16 expression vector. Results of the Transwell assay demonstrated that enforced P16 overexpression significantly reversed the enhanced migration phenotype of these cells (Fig. 7a). Similar results were also observed in HONE-1 cells (Additional file 1: Figure S6). In contrast, downregulation of endogenous P16 expression through transient siRNA transfection significantly enhanced the migration of BGC823 and GES-1 cells (Fig. 7b). Furthermore, the migration capacity of A549 cells, which lack P16 alleles, was not changed following stable transfection of P16-dnmt and 7 days of doxycycline treatment (Fig. 7c). Taken together, these results imply that the enhanced migration and invasion phenotypes of cancer cells are P16-specific.
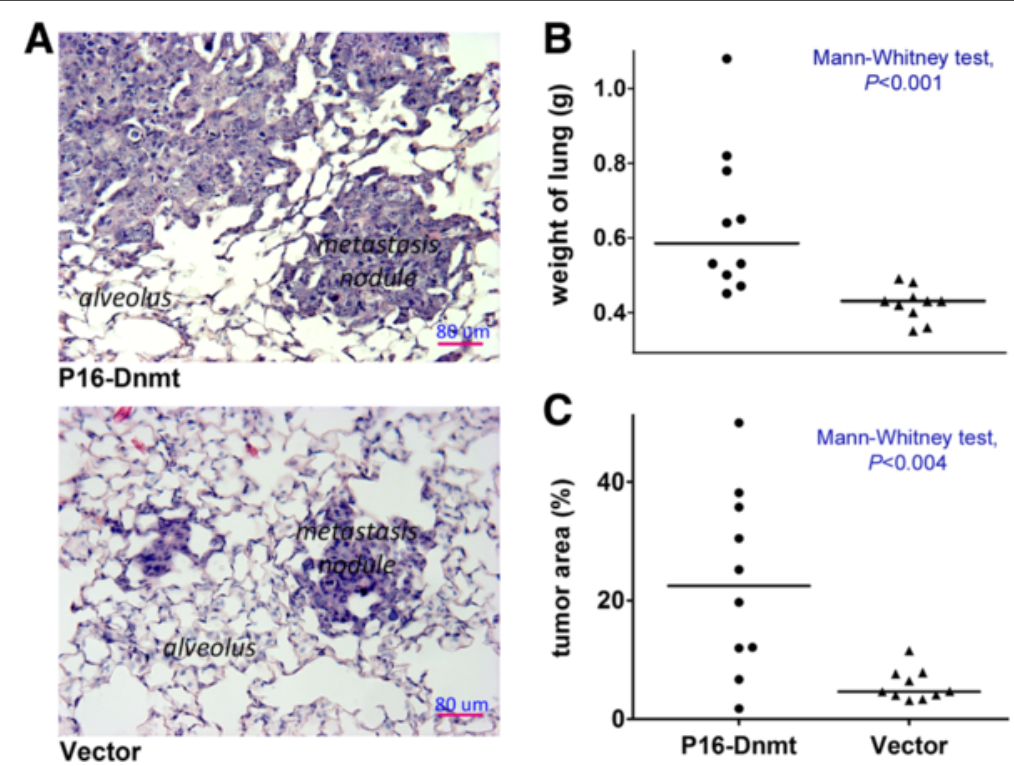

Fig. 6 P16-specific methylation promotes experimental pneumonic metastasis of BGC823 cells. a Images of representative metastatic nodules in the lung of SCID mice (H\&E staining). b The lung weights of mice in the P16-dnmt PTRIPZ and control groups at day 19. c The ratio of metastatic nodule area to lung area of mice in the P16-dnmt pTRIPZ and control groups 


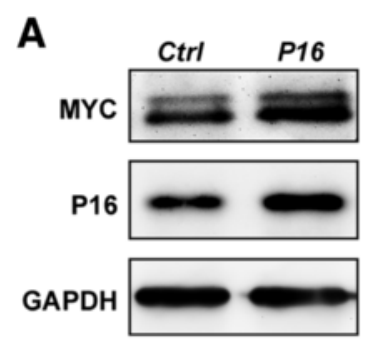

B
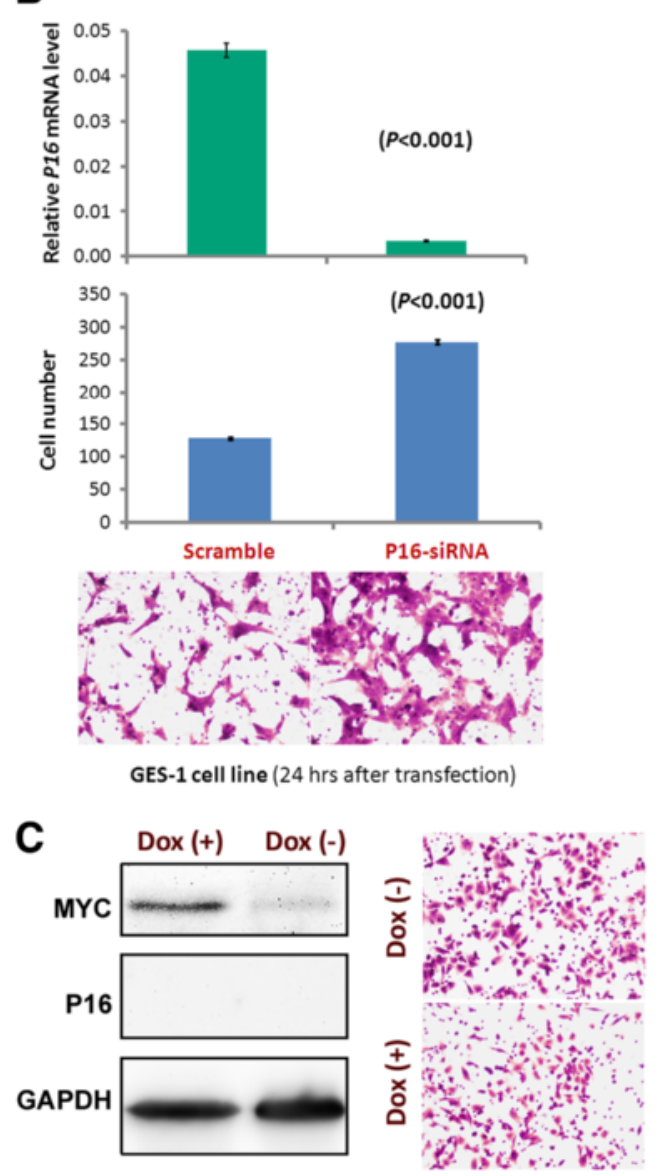
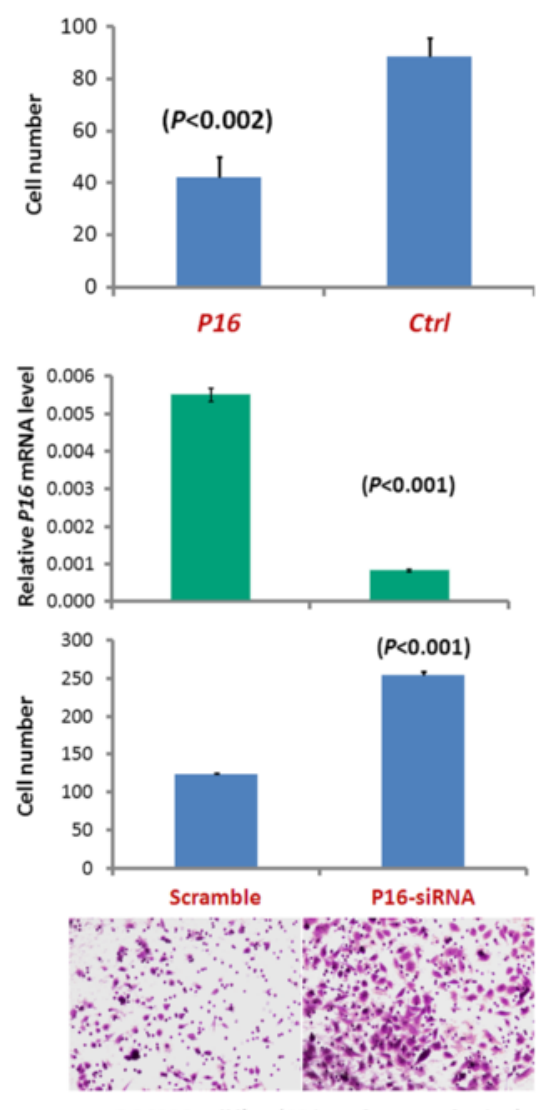

BGC823 cell line ( $48 \mathrm{hrs}$ after transfection)

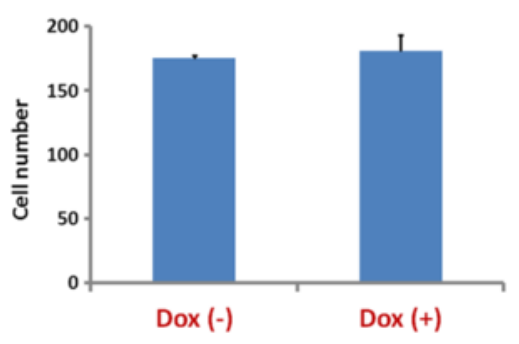

Fig. 7 Effect of varying P16 expression on migration. a Rescue assay demonstrating the effect of enforced P16 overexpression on migration of the P16-dnmt stably transfected BGC823 cells treated with doxycycline for 2 weeks. b Migration assay results after 24-h and 48-h transient siRNA transfection in GES-1 and BGC823 cell lines. P16 qRT-PCR results are also presented. c Migration capacity of A549 cells (lacking P16 alleles) following stable transfection of P16-dnmt. After treatment with $0.25 \mathrm{\mu g} / \mathrm{mL}$ doxycycline for 1 week, these cells $\left(4.0 \times 10^{4}\right)$ were seeded into each well and incubated for $28 \mathrm{~h}$. Western blot analysis of P16-Dnmt and P16 expression is also presented. Migration assays were independently repeated in triplicate

P16-specific DNA methylation promotes RB phosphorylation and upregulates NFKB subunit P65 expression

To confirm that P16 DNA methylation affects its downstream signal pathway, P16-CDK4/6-RB, the phosphorylation level of RB protein was analyzed using Western blot analysis. As expected, increased levels of phosphorylated RB were detected in the P16-dnmt transfected BGC823 and GES-1 cells treated with doxycycline when compared to those without doxycycline induction and those transfected with the control vector. Total RB protein levels were not changed (Fig. 8a and b). Furthermore, the expression level of nuclear factor NFkB subunit P65 was also increased in the P16-dnmt transfected cells.

\section{Discussion}

It is well known that methylation of $\mathrm{CpG}$ islands around transcription start sites is inversely correlated with the 


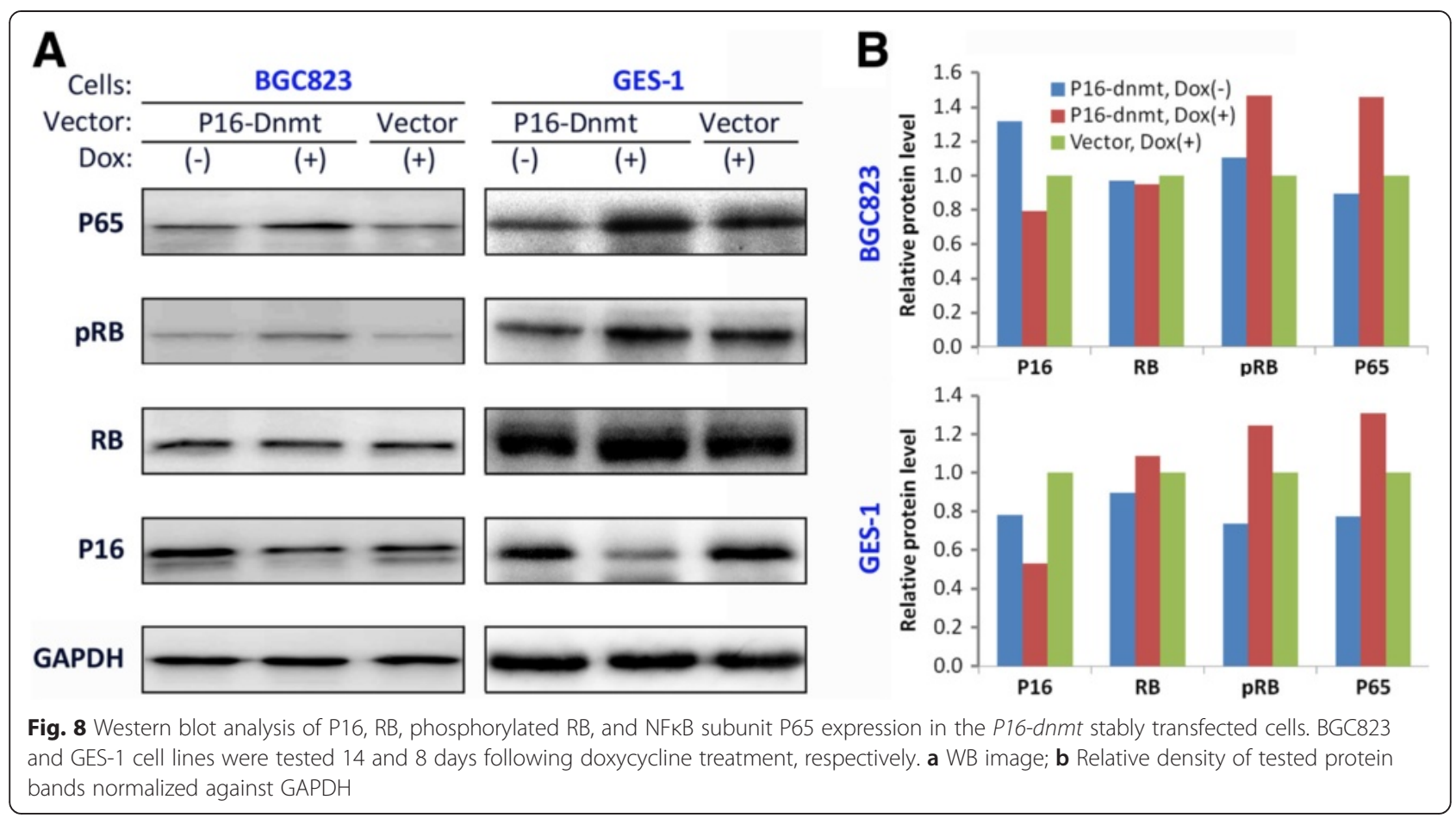

expression level of genes in many cells and tissues. P16 DNA methylation may occur as a long-term mechanism to maintain gene suppression following transcriptional silence induced by repressive histone modifications [17]. Whether P16 promoter methylation alone is capable of silencing transcription has not been well studied. In the present study, we found that P16-Dnmt-induced methylation of P16 CpG islands could directly inactivate gene expression and promote metastasis of cancer cells.

Studies have shown that target-specific methylation/ demethylation is associated with transcriptional inactivation/re-activation of several other human genes [18-20]. Zhang et al. reported that P16-specific artificial transcription factor (P16-ATF) could induce P16 DNA demethylation and re-activate its expression [21]; however, it is not known whether DNA demethylation is essential for re-activation of this gene. Yu et al. successfully established a $p 16$ DNA methylation model in mice through the insertion of alu motifs into the mouse $p 16$ promoter and found subsequent gene inactivation [16]; however, the possibility that the insertion of alu motifs directly contributed to transcriptional repression cannot be excluded. In order to increase the targeting specificity in the present study, we employed the pTRIPZ vector that allowed for controllable expression of P16-Dnmt. Our results showed that P16-Dnmt-induced DNA methylation was sufficient to silence transcription in two human gastric epithelial cell lines. This result is consistent with a recent report which demonstrated that engineered transcription activator-like effector (TALE)-Dnmts induced
P16 DNA methylation, inactivated gene expression, and increased replication in human fibroblasts [22]. Additionally, steric hindrance from P16-Dnmt DNA binding does not appear to play a significant role in repressing gene transcription as was demonstrated using the P16-dnmt R882H mutant control. Taken together, the evidence suggests that P16 DNA methylation primarily accounts for the inactivation of P16 transcription.

Genome-wide CRISPR screens in mouse models have shown that genetic $p 16$ inactivation may be a driver for tumor growth and metastasis [5]. Luo et al. have reported that the proportion of methylated P16 alleles is significantly associated with metastasis of gastric cancers [13]. Zhang et al. have also suggested that re-activation of methylated P16 by P16-ATF inhibits migration and invasion in AGS and H1299 cancer cell lines [21]. Here, we have provided evidence to demonstrate that P16-Dnmt mediated DNA methylation might promote metastasis of cancer cells in vitro and in vivo. Moreover, we found that such an effect was not observed in A549 cells lacking P16 alleles, and siRNA downregulation of $P 16$ expression also promoted the migration of cell lines, and overexpression of $P 16$ reversed the cell migration phenotype. These facts strongly implicate inactivation of P16 by DNA methylation as a possible promoter of migration/invasion and metastasis of cancer cells.

Inactivation of the P16 gene results in higher cyclin D-dependent protein kinase activity and thus induces aberrant phosphorylation of RB protein. Therefore normal cell cycle checkpoints are bypassed allowing accelerated 
cell growth and increased genomic instability [23, 24]. We found that the induction of P16-specific DNA methylation could also increase the phosphorylation of RB.

$\mathrm{NF \kappa B}$ subunit P65 is the master regulator in the senescence-associated secretary phenotype (SASP) [25]. In melanomas, the expression of P65 is increased while P16 expression is decreased [26]. P65 also regulates the transcription of a group of metastasis-related genes, including MMP-9/2 [27-29]. In this study, we also found that induction of P16 DNA methylation also increases the amount of P65 protein in cancer cells. Additional studies are required to determine other pathways involved in the P16 DNA methylation-related metastasis phenotype.

\section{Conclusion}

Engineered zinc finger protein-targeted P16 DNA methylation directly inactivates P16 expression and promotes invasion and metastasis of cancer cells.

\section{Methods}

Cell lines and cultures

HEK293T, BGC823, and GES-1 cell lines were kindly provided by Professor Yang Ke at Peking University Cancer Hospital and Institute. The A549 cell line lacking the P16 locus was kindly provided by Professor Zhiqian Zhang at the same institute. The HONE-1 cell line was kindly provided by Professor Zhen Sun at Capital Medical University School of Stomatology, Beijing. All of these cell lines were tested and authenticated using the Goldeneye20A STR Identifiler PCR Amplification Kit (Beijing Jianlian Genes Technology Co., Ltd.) before being used in this study [30]. These cell lines were cultured in RPMI1640 medium supplemented with $10 \% \mathrm{FBS}$ and maintained at $37{ }^{\circ} \mathrm{C}$ in humidified air with $5 \% \mathrm{CO}_{2}$. Cell proliferation was analyzed using the Cell Counting Kit-8 (CCK-8) [21].

\section{Construction of vectors and transfection}

The P16-dnmt plasmid was constructed by fusing a SP1-like engineered seven-zinc finger protein (7ZFP) $6 I$ capable of specifically binding the 21-bp fragment (5'-GAG GAA GGA AAC GGG GCG GGG-3', including a Sp1-binding site) within the human P16 promoter [21] with the catalytic domain (approximately 608-908aa) of mouse dnmt3a in the pFast Bac HT A-dnmt3a vector (kindly provided by Professor Keith Robertson at Georgia Regents University, USA) [31]. Point mutation $\mathrm{R} 882 \mathrm{H}$ in the catalytic domain of Dnmt3a is the most frequent somatic mutation in acute myeloid leukemia [32]. The methyltransferase activity of R882H DNMT3A is reduced by approximately $80 \%$ compared with the wide-type [33]. Thus, a P16-dnmt R882H mutant control was constructed as a negative control. The P16-dnmt coding sequence was integrated into a pcDNA3.1 vector and an expression controllable pTRIPZ vector carrying a 'Tet-on' switch (Open Biosystem, USA), respectively. Control vectors for the Dnmt3a catalytic domain or 7ZFP(6I) were also constructed. The purified P16-dnmt plasmid DNA was mixed with VSVG and $\Delta 8.9$ (Addgene, USA) to prepare lentivirus transfection particles. The P16 expression vector was constructed using wild-type P16 coding sequence cDNA and integrated into the pIRES2-EGFP vector. The cells $\left(4.5 \times 10^{4}\right)$ were transiently transfected with the pIRES2-P16 expression vector, seeded into each well, and incubated for 43 h. P16 specific siRNAs (5'CCGUA AAUGU CCAUU UAUAT T-3' and 5'-UAUAA AUGGA CAUUU ACGGT T-3') were synthesized (GenePharma) and used to transiently transfect cells at a final concentration of $1.0 \mu \mathrm{g} / 1 \mathrm{~mL}$. The scramble siRNAs (5'-UUCUC CGAAC GUGUC ACGUT T-3' and 5'-ACGUG ACACG UUCGG AGAAT T-3') were used as negative control. The fresh lentivirus particles were used to transfect human cells.

\section{Bisulfite-DHPLC, -sequencing, MethyLight, and methylation-specific PCR (MSP)}

The 392-bp fragments isolated from the antisense-strand of P16 exon-1 in cultured cells were amplified with a CpG-free primer set and analyzed using DHPLC and clone sequencing as described previously [13, 34]; however, the PCR annealing temperature was fixed at $57.0{ }^{\circ} \mathrm{C}$ to avoid amplification bias between methylated and unmethylated P16 alleles. The 567-bp fragment in the antisense-strand of the $P 16$ promoter was also amplified using a CpG-free primer set (forward, $5^{\prime}$-gaatt agggt ttttg attta gtgaa $\mathrm{tt}-3^{\prime}$; reverse, $5^{\prime}$-accct atccc tcaaa tcctc taaa- $3^{\prime}$ ) at an annealing temperature of $65{ }^{\circ} \mathrm{C}$, analyzed at the partial denaturing temperature of $54{ }^{\circ} \mathrm{C}$ in DHPLC analysis, and confirmed using clone sequencing. Methylated and unmethylated P16 were also analyzed by 150/151-bp MSP [35].

The 272-bp P14 CpG island fragment was amplified using a CpG-free primer set (forward, $5^{\prime}$-gttgt ttatt tttgg tgtta-3'; reverse, $5^{\prime}$-acctt tccta cctaa tcttc- $3^{\prime}$ ) at the annealing temperature of $51.0{ }^{\circ} \mathrm{C}$, and analyzed at the partial denaturing temperature of $57.7{ }^{\circ} \mathrm{C}$ in DHPLC analysis. The 437-bp ZNF382 CpG island fragment was amplified and analyzed by DHPLC as previously described [30].

\section{Quantitative RT-PCR, Western blot, and confocal analysis of $P 16$ expression}

The P16 mRNA and protein level in cell lines were analyzed as described [21]. 


\section{Chromatin immunoprecipitation (ChIP) assays}

The 124-bp P16 and 61-bp P14 DNA fragments within CpG islands bound to P16-Dnmt were quantitated as described [21, 36]. Anti-Myc antibody was used to precipitate P16-Dnmt protein containing a Myc tag. The Myc-ChIPed-DNA samples were sequenced using the Illumina HiSeq2500 (Shanghai Biotechnology Co., China). The readouts were preprocessed using online fastx software (version 0.0.13; http://hannonlab.schl.edu/fastx_toolkit/ index.html), mapped to the human genome hg19 using Bowties (version o.12.8) [37], and enriched using MACS (version 1.4.2) [38]. The protein-binding motif was identified using MEME software [39]. The detected peaks/annotated information is presented as Additional file 2: File S1, Additional file 3: File S2, and Additional file 4: File S3.

\section{Genome-wide analysis of DNA methylation}

Illumina Infinium HD Methylation450K arrays were used to perform differential CpG methylation analyses on BGC823 cells stably transfected with the P16-dnmt and pTRIPZ control vectors following 14 days of doxycycline treatment according to the Assay Manual. Two parallel samples were tested for each group. DNA methylation levels for each CpG site were computed as the ratio of normalized methylated signal intensity to the sum of methylated and unmethylated signal intensities using GenomeStudio software. Using the control vector as a reference, $\Delta \beta$ was calculated to represent differential methylation for each CpG site in the P16-dnmt expressing cells. The differential methylation was considered to be significant when the $\Delta \beta$ value was $>0.50$. The raw methylation data are available as Additional file 6: File S5.

\section{Dual-luciferase reporter assay}

The $P 16$ promoter (approximately -597 to $+155 \mathrm{nt}$ ) was integrated into the pGL3-Basic vector and used for promoter activity analysis as previously described [21].

\section{Transwell migration and Matrigel invasion tests}

The Transwell migration and Matrigel invasion tests were performed using GES-1 and BGC823 cells suspended in $150 \mu \mathrm{L}$ serum-free medium $\left(2 \times 10^{5}\right.$ cells/ $\mathrm{mL})$. The BGC823 cells were incubated for $36 \mathrm{~h}$ and 96 $\mathrm{h}$ at $37{ }^{\circ} \mathrm{C}$ in $5 \% \mathrm{CO}_{2}$ before quantifying their migration and invasion capacity, respectively. Similarly, the GES-1 cells were quantified following 48-h and 108-h incubation, respectively [21]. Wound healing status was dynamically recorded using the IncuCyte $\mathrm{ZOOM}^{\mathrm{Tm}}$ livecell imaging platform. Each trial consisted of three independent samples, and all the assays were repeated two to three times.
Xenografts and pneumonic metastases in SCID mice

GES-1 cells $\left(1.4 \times 10^{6}\right.$ cells in $200 \mu \mathrm{L}$ Matrigel suspension) were stably transfected with the P16-Dnmt or control vector, induced with $0.25 \mu \mathrm{g} / \mathrm{mL}$ doxycycline for 7 days, and then injected subcutaneously into the lower limb of NOD SCID mice (female, 5 weeks old, weight 10-20 g, purchased from Beijing Huafukang Biotech). The mice were provided distilled, sterile water containing $2 \mu \mathrm{g} / \mathrm{mL}$ doxycycline. These mice were sacrificed 48 days after transplantation. The weight and volume of tumors were then analyzed.

For the pneumonic metastasis assay, BGC823 cells stably transfected with the P16-dnmt or control vector were also induced with $0.25 \mu \mathrm{g} / \mathrm{mL}$ doxycycline for 7 days, and then injected into the tail vein of the SCID mice $\left(1.5 \times 10^{6}\right.$ cells in $0.15 \mathrm{~mL}$ medium) (10 randomly allocated mice per group). The lung weight was detected at the 19th experimental day for each mouse [40]. The lung organs were fixed with Bouin solution, paraffinembedded and cut into $5 \mu \mathrm{m}$ slides along the maximum area, and examined microscopically following $\mathrm{H} \& \mathrm{E}$ staining. The lung area and total tumor nodule area were measured using INFINITY Analyze (Version 4.0, Lumenera Sci). The nodule area to the lung area ratio was calculated for each mouse.

\section{Statistical analysis}

Results were displayed by constituent ratios of enumeration or ranked data. All $P$ values were two-sided, and a difference with $P<0.05$ was considered statistically significant.

\section{Ethical approval}

This study was approved by the institute's animal ethics committee (approval no. AE-2012-06).

\section{Data and materials availability}

The methylation array data have been deposited into the Gene Expression Omnibus under accession number GSE74233. The ChIP-sequencing raw data have been deposited into the bioproject database under accession number SRA306603.

\section{Additional files}

Additional file 1: Figures S1 to S6. (DOC $3228 \mathrm{~kb}$ )

Additional file 2: File S1 (annotated_tss_P16Dnmt-

Myc.fq_macs_peaksFile). (XLSX 1943 kb)

Additional file 3: File S2 (annotated_tss_P16Dnmt-

IgG.fq_macs_peaks). (XLSX 139 kb)

Additional file 4: File S3 (annotated_tss_vector-

Myc.fq_macs_peaks). (XLSX 153 kb)

Additional file 5: File S4 (P16-Dnmt induced methylation sites). (XLSX 4152 kb)

Additional file 6: File S5 (450 K RawSignal). (CSV 46821 kb) 


\section{Abbreviations}

7ZFP: seven zinc finger protein; DHPLC: denatured high performance liquid chromatography; Dox: doxycycline; P16-ATF: P16-specific transcription factor P16-Dnmt: P16-specific methyltransferase.

\section{Competing interests}

The authors declare that they have no competing interests.

\section{Authors' contributions}

CC and YG carried out the cellular and molecular biological studies, participated in the design of the study and construction of various expression vectors, and drafted the manuscript. LG carried out the confocal immunoassays. JW edited the manuscript. ZL participated in methylation analysis by DHPLC. BZ and DD conceived of the study, participated in its design and coordination, and helped to draft the manuscript. All authors read and approved the final manuscript.

\section{Acknowledgements}

The abstract has been reported at the 4th International Cancer Epigenetics Meeting on October 17-20, 2014, Yangzhou, China.

\section{Funding}

This work was supported by the National Basic Research Programs of China to D.D. (nos. 2011 CB504201 and 2015CB553902), a grant from the National Nature Science Foundation of China A3 Foresight Program to D.D. (no. 31261140372), and a grant from Beijing Science and Technology Commission to D.D. (no. Z151100001615022)

\section{Author details}

${ }^{1}$ Key Laboratory of Carcinogenesis and Translational Research (Ministry of Education/Beijing), Department of Aetiology, Peking University Cancer Hospital \& Institute, Beijing 100142, China. '2Department of Pathology, Institute of Hematology \& Hospital of Blood Diseases, Chinese Academy of Medical Sciences, Tianjin 300020, China. ${ }^{3}$ GRU Cancer Center, Georgia Regents University, Augusta GA30912, USA

\section{Received: 30 September 2015 Accepted: 30 October 2015} Published online: 23 November 2015

\section{References}

1. Beroukhim R, Mermel CH, Porter D, Wei G, Raychaudhuri S, Donovan J, et al. The landscape of somatic copy-number alteration across human cancers. Nature. 2010;463:899-905.

2. Liu L, Lassam NJ, Slingerland JM, Bailey D, Cole D, Jenkins R, et al. Germline p16INK4A mutation and protein dysfunction in a family with inherited melanoma. Oncogene. 1995;11:405-12.

3. Hussussian CJ, Struewing JP, Goldstein AM, Higgins PA, Ally DS, Sheahan MD, et al. Germline p16 mutations in familial melanoma. Nat Genet. 1994:8:15-21.

4. Kannengiesser C, Brookes S, del Arroyo AG, Pham D, Bombled J, Barrois M, et al. Functional, structural, and genetic evaluation of 20 CDKN2A germ line mutations identified in melanoma-prone families or patients. Hum Mutat. 2009;30:564-74.

5. Chen S, Sanjana NE, Zheng K, Shalem O, Lee K, Shi X, et al. Genome-wide CRISPR screen in a mouse model of tumor growth and metastasis. Cell. 2015:160:1246-60.

6. Merlo A, Herman JG, Mao L, Lee DJ, Gabrielson E, Burger PC, et al. 5' CpG island methylation is associated with transcriptional silencing of the tumour suppressor p16/CDKN2/MTS1 in human cancers. Nat Med. 1995;1:686-92.

7. Serrano J, Goebel SU, Peghini PL, Lubensky IA, Gibril F, Jensen RT. Alterations in the p16INK4a/CDKN2A tumor suppressor gene in gastrinomas. J Clin Endocrinol Metab. 2000;85:4146-56.

8. Herman JG, Merlo A, Mao L, Lapidus RG, Issa JPJ, Davidson NE, et al. Inactivation of the Cdkn2/P16/Mts1 gene is frequently associated with aberrant DNA methylation in all common human cancers. Cancer Res. 1995; 55:4525-30.

9. Gonzalez-Zulueta M, Bender CM, Yang AS, Nguyen T, Beart RW, Van Tornout $\mathrm{JM}$, et al. Methylation of the $5^{\prime} \mathrm{CpG}$ island of the p16/CDKN2 tumor suppressor gene in normal and transformed human tissues correlates with gene silencing. Cancer Res. 1995;55:4531-5.
10. Kresty LA, Mallery SR, Knobloch TJ, Song HJ, Lloyd M, Casto BC, et al. Alterations of p16(INK4a) and p14(ARF) in patients with severe oral epithelial dysplasia. Cancer Res. 2002;62:5295-300.

11. Sun Y, Deng DJ, You WC, Bai H, Zhang L, Zhou J, et al. Methylation of p16 CpG islands associated with malignant transformation of gastric dysplasia in a population-based study. Clin Cancer Res. 2004;10:5087-93.

12. Belinsky SA, Liechty KC, Gentry FD, Wolf HJ, Rogers J, Vu K, et al. Promoter hypermethylation of multiple genes in sputum precedes lung cancer incidence in a high-risk cohort. Cancer Res. 2006;66:3338-44.

13. Luo DY, Zhang BZ, Lv LB, Xiang SY, Liu YH, Ji JF, et al. Methylation of CpG islands of p16 associated with progression of primary gastric carcinomas. Lab Investig. 2006;86:591-8.

14. Cao J, Zhou J, Gao Y, Gu L, Meng H, Liu H, et al. Methylation of p16 CpG island associated with malignant progression of oral epithelial dysplasia: a prospective cohort study. Clin Cancer Res. 2009;15:5178-83.

15. Liu HW, Liu XW, Dong GY, Zhou J, Liu Y, Gao Y, et al. P16 methylation as an early predictor for cancer development from oral epithelial dysplasia: a double-blind multicentre prospective study. EBioMedicine. 2015;2:432-6.

16. Yu DH, Waterland RA, Zhang P, Schady D, Chen MH, Guan Y, et al. Targeted p16(Ink4a) epimutation causes tumorigenesis and reduces survival in mice. J Clin Invest. 2014;124:3708-12.

17. Hinshelwood RA, Melki JR, Huschtscha LI, Paul C, Song JZ, Stirzaker C, et al. Aberrant de novo methylation of the p16INK4A CpG island is initiated post gene silencing in association with chromatin remodelling and mimics nucleosome positioning. Hum Mol Genet. 2009;18:3098-109.

18. Maeder ML, Angstman JF, Richardson ME, Linder SJ, Cascio VM, Tsai SQ, et al. Targeted DNA demethylation and activation of endogenous genes using programmable TALE-TET1 fusion proteins. Nat Biotechnol. 2013;31: 1137-42.

19. Chen H, Kazemier HG, de Groote ML, Ruiters MH, Xu GL, Rots MG. Induced DNA demethylation by targeting Ten-Eleven Translocation 2 to the human ICAM-1 promoter. Nucleic Acids Res. 2014;42:1563-74.

20. Rivenbark AG, Stolzenburg S, Beltran AS, Yuan X, Rots MG, Strahl BD, et al. Epigenetic reprogramming of cancer cells via targeted DNA methylation. Epigenetics. 2012;7:350-60.

21. Zhang B, Xiang S, Zhong Q, Yin Y, Gu L, Deng D. The p16-specific reactivation and inhibition of cell migration through demethylation of $\mathrm{CpG}$ islands by engineered transcription factors. Hum Gene Ther 2012;23:1071-81.

22. Bernstein DL, Le Lay JE, Ruano EG, Kaestner KH. TALE-mediated epigenetic suppression of CDKN2A increases replication in human fibroblasts. J Clin Invest. 2015;125:1998-2006

23. Serrano M, Hannon GJ, Beach D. A new regulatory motif in cell-cycle control causing specific inhibition of cyclin D/CDK4. Nature. 1993:366:704-7.

24. McDermott KM, Zhang J, Holst CR, Kozakiewicz BK, Singla V, Tlsty TD p16(INK4a) prevents centrosome dysfunction and genomic instability in primary cells. PLoS Biol. 2006;4, e51.

25. Chien Y, Scuoppo C, Wang X, Fang X, Balgley B, Bolden JE, et al. Control of the senescence-associated secretory phenotype by NF-kB promotes senescence and enhances chemosensitivity. Genes Dev. 2011;25:2125-36.

26. Ghiorzo P, Mantelli M, Gargiulo S, Gramigni C, Pastorino L, Banelli B, et al. Inverse correlation between p16INK4A expression and NF-kappaB activation in melanoma progression. Hum Pathol. 2004;35:1029-37.

27. Song ZB, Ni JS, Wu P, Bao YL, Liu T, Li M, et al. Testes-specific protease 50 promotes cell invasion and metastasis by increasing NF-kappaB-dependent matrix metalloproteinase-9 expression. Cell Death Dis. 2015;6, e1703.

28. Li HL, Han L, Chen HR, Meng F, Liu QH, Pan ZQ, et al. PinX1 serves as a potential prognostic indicator for clear cell renal cell carcinoma and inhibits its invasion and metastasis by suppressing MMP-2 via NF-kB-dependent transcription. Oncotarget. 2015;6:21406-20.

29. Zhang $Y$, Huang $H$, Zhou H, Du T, Zeng $L$, Cao $Y$, et al. Activation of nuclear factor $\mathrm{KB}$ pathway and downstream targets survivin and livin by SHARPIN contributes to the progression and metastasis of prostate cancer. Cancer. 2014;120:3208-18.

30. Liu ZJ, Zhang J, Gao YH, Pei LR, Zhou J, Gu LK, et al. Large-scale characterization of DNA methylation changes in human gastric carcinomas with and without metastasis. Clin Cancer Res. 2014;20:4598-612.

31. Van Emburgh BO, Robertson KD. Modulation of Dnmt3b function in vitro by interactions with Dnmt3L, Dnmt3a and Dnmt3b splice variants. Nucleic Acids Res. 2011;39:4984-5002. 
32. Xu J, Wang YY, Dai YJ, Zhang W, Zhang WN, Xiong SM, et al. DNMT3A Arg882 mutation drives chronic myelomonocytic leukemia through disturbing gene expression/DNA methylation in hematopoietic cells. Proc Natl Acad Sci U S A. 2014;111:2620-5.

33. Russler-Germain DA, Spencer DH, Young MA, Lamprecht TL, Miller CL, Fulton $\mathrm{R}$, et al. The R882H DNMT3A mutation associated with AML dominantly inhibits wild-type DNMT3A by blocking its ability to form active tetramers. Cancer Cell. 2014;25:442-54.

34. Deng DJ, Deng GR, Smith MF, Zhou J, Xin HJ, Powell SM, et al. Simultaneous detection of $\mathrm{CpG}$ methylation and single nucleotide polymorphism by denaturing high performance liquid chromatography Nucleic Acids Res. 2002;30:13E.

35. Herman JG, Graff JR, Myohanen S, Nelkin BD, Baylin S. Methylation-specific PCR: a novel PCR assay for methylation status of $\mathrm{CpG}$ islands. Proc Natl Acad Sci U S A. 1996;93:9821-6.

36. Li Q, Wang X, Lu Z, Zhang B, Guan Z, Liu Z, et al. Polycomb CBX7 directly controls trimethylation of histone $\mathrm{H} 3$ at lysine 9 at the p16 locus. PLoS One. 2010;5, e13732.

37. Langmead B, Trapnell C, Pop M, Salzberg SL. Ultrafast and memory-efficient alignment of short DNA sequences to the human genome. Genome Biol. 2009;10:R25.

38. Zhang Y, Liu T, Meyer CA, Eeckhoute J, Johnson DS, Bernstein BE, et al. Model-based Analysis of ChIP-Seq (MACS). Genome Biol. 2008;9:R137.

39. Machanick P, Bailey TL. MEME-ChIP: motif analysis of large DNA datasets. Bioinformatics. 2011;2712:1696-7.

40. Kijima-Suda I, Miyamoto Y, Toyoshima S, Itoh M, Osawa T. Inhibition of experimental pulmonary metastasis of mouse colon adenocarcinoma 26 sublines by a sialic acid:nucleoside conjugate having sialyltransferase inhibiting activity. Cancer Res. 1986;46:858-62.

\section{Submit your next manuscript to BioMed Central and take full advantage of:}

- Convenient online submission

- Thorough peer review

- No space constraints or color figure charges

- Immediate publication on acceptance

- Inclusion in PubMed, CAS, Scopus and Google Scholar

- Research which is freely available for redistribution 